\title{
Luminance as a factor in the ability of achromatic gratings to interfere with McCollough effects
}

\author{
D. SKOWBO \\ Colby College, Waterville, Maine 04901
}

\begin{abstract}
McCollough effects were reduced in assessed strength by having observers view achromatic gratings that varied in space-average luminance. Whether the gratings were presented before or after induction of McCollough effects, high luminance gratings interfered with the effects more than did low luminance gratings. If considered within the context of a conditioning model, this finding may be interpreted as inconsistent with other characteristics of McCollough effects.
\end{abstract}

Aftereffects of color which are contingent upon the orientation of lines were first reported in 1965 by McCollough. Particularly because the subjective coloration has an unusually long duration (see, for example, Jones \& Holding, 1975), these aftereffects have been the subject of considerable theoretical debate. Accounts of how the visual system might produce and maintain McCollough effects (MEs) have fallen into two general classes. One type of model proposes that MEs result from adaptation in neural channels sensitive to wavelength and orientation (e.g., Held \& Shattuck, 1971). According to another kind of model, MEs arise from the classical conditioning of an adaptive color response to the presentation of lines (Murch, 1976). The fact that exposure to achromatic lines hastens the decay of MEs (Skowbo, Gentry, Timney, \& Morant, 1974; White \& Graves, Note 1) has been taken as support for the latter model: Presentations of achromatic patterns would be comparable to the presentations of a conditioned stimulus in the absence of the unconditioned stimulus, and hence would tend to extinguish the conditioned response.

However, there are two alternative ways of accounting for the interfering effect of achromatic lines, both of which are consistent with adaptation models of MEs. One involves postulating the existence of an achromatic analogue to MEs, biasing the visual system towards neutrality, rather than chromaticity. If this kind of response tendency were introduced, it might be expected to compete with a previously established chromatic effect, reducing its apparent strength (Skowbo \& Clynes, 1977; White, Note 2). Another interpretation of the interference effect is that viewing achromatic gratings may diminish the apparent contrast of subsequently presented test patterns. White and Graves (Note 3) suggested this ex-

This research was supported by a Social Sciences Research Grant from Colby College. planation on the basis of their finding that lowered test pattern contrast reduces the efficiency of testing MEs.

The extent to which viewing achromatic gratings can diminish strength indexes has been studied parametrically with regard to time of exposure (Skowbo \& Clynes, 1977), but a variable of particular theoretical interest is the intensity of the gratings. Luminance has been found to be extremely important in the establishment and testing of MEs: White (1976) has shown that the apparent magnitude of MEs is greater following high-luminance inspection patterns, and on test patterns of relatively low luminance. Therefore, one might reason that if a competing achromatic effect were being established by viewing black and white gratings, a high-luminance pattern should interfere with the chromatic phenomenon more than a low-luminance pattern. A high-luminance pattern would also be expected to cause a greater reduction in apparent contrast. On the other hand, if the interference phenomenon is the result of disrupting a conditioned association between lines and color, then the effect might be expected to weaken most when the luminance of the conditioned stimulus approximates that which elicits the strongest response; i.e., lowluminance gratings would produce maximal interference (see also Discussion section).

Another property of achromatic gratings is their ability to interfere with MEs in advance of their induction (White \& Graves, Note 1). Chromatic effects induced after viewing achromatic gratings are weaker than typical. This phenomenon can be encompassed by an adaptation model as the generation of an achromatic effect which later competes with the chromatic effect; and by a learning model as latent inhibition. It has also not been studied with regard to intensity.

The present study is an attempt to assess the ability of achromatic gratings of different luminances to interfere with apparent magnitude of MEs. 


\section{METHOD}

Three trained observers with normal color vision were used. One was familiar with the theoretical background of the study; the other two were not. Observers were seated facing two adjacent circular fields $11^{\circ}$ in diameter on a black background. Adaptation patterns were projected onto the left field. MEs were assessed by having observers match effects seen on a test grating, projected onto the left-hand field, with chromatic adjustments of a homogeneous surface projected onto the right-hand field. The chromatic stimulus was provided by a projection colorimeter which mixed lights transmitted by 2 Wratten filters (CC30M and CC50G). All matches could be located in CIE space along a straight line connecting two points with the coordinates $x=.407, y=.454$ and $\mathrm{x}=.421, \mathrm{y}=.350$.

In Experiment 1, each session began with $5 \mathrm{~min}$ of adaptation to two achromatic fields $\left(8.2 \mathrm{~cd} / \mathrm{m}^{2}\right)$, followed by six matches each, in ABBA sequence, to the vertical and horizontal portions of the test pattern. Subsequently, MEs were induced by having the observer view alternating vertical or horizontal green (Wratten 53 ) or magenta (Wratten 32) gratings ( 5 cycles/deg). The gratings changed every $10 \mathrm{sec}$ for a total of $5 \mathrm{~min}$. After another $5 \mathrm{~min}$ of light adaptation, the observer again matched the two portions of the display. Ten minutes of exposure to alternating achromatic horizontal and vertical gratings followed. These gratings were one of three space-average luminances: $6.8 \mathrm{~cd} / \mathrm{m}^{2}$ (same as test pattern luminance), 48.0 , or $480 \mathrm{~cd} / \mathrm{m}^{2}$. Finally, after another $5-\mathrm{min}$ period of light adaptation, the test pattern was matched once again.

In Experiment 2, the observer also began with a match to the test pattern; he or she then viewed alternating horizontal and vertical achromatic gratings for $10 \mathrm{~min}$. Luminances were the same as in Experiment 1. Observers sat for 2 min with closed eyes, then inspected alternating chromatic gratings for $5 \mathrm{~min}$, as in Experiment 1. After $5 \mathrm{~min}$ of light adaptation, a second set of matches to the test pattern was obtained.

In both experiments, each observer participated in nine sessions (three at each luminance). At least 48 h elapsed between sessions, and the color-orientation combination used to induce chromatic effects was reversed in successive sessions.

\section{RESULTS}

For all matching blocks, the mean of six matches to each half of the test pattern was converted to CIE coordinates. The dependent variable, an index of strength of the effect, was taken as the distance in CIE space between the locations of the two individual matches. This distance was minimal (typically, near zero) for the preadaptation matches and maximal for matches made just after chromatic adaptation.

To summarize the data obtained in Experiment 1, the distance between matches made after viewing achromatic gratings was divided by the distance obtained after inspecting chromatic patterns (the initial strength of the effect). This normalization of data provided a percentage of the initial ME remaining after achromatic patterns had been viewed.

Figure 1 represents this percent remaining, averaged across sessions and observers. The trend indicated by the height of the bars is that inspecting high-luminance patterns leads to the most reduction in the strength index, while viewing low-luminance patterns brings about the least reduction.

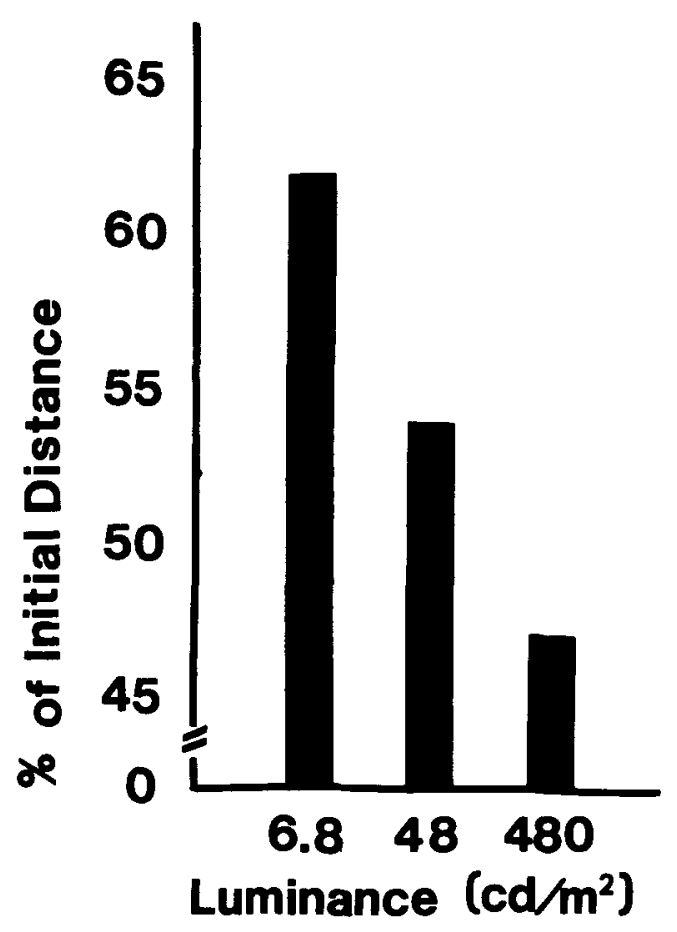

Figure 1. Amount of initial $\mathrm{ME}$ remaining after exposure to achromatic gratings of varying space-average luminance. MEs are assessed as distance in CIE space between matches to two parts of a test pattern. The height of each bar represents an average of three sessions for each of three observers.

In Experiment 2, a distance between matches was also obtained. This distance indicated strength of a freshly induced chromatic effect, but the effect was induced after the observers had viewed achromatic patterns. For comparison purposes, an average of initial-strength indexes across all conditions in Experiment 1 was obtained for each observer. This figure was divided into the distances obtained in Experiment 2; the result provides an estimate of the extent to which each observer showed effects that were weaker than typical.

Figure 2 shows percent of typical initial strength obtained by inducing MEs after achromatic inspections. Differences among the conditions are smaller than in Experiment 1, and only the high-luminance condition appears to be different from the other two conditions.

\section{DISCUSSION}

The results of the present study make it possible to reevaluate the several possible explanations of the interference effect. The findings of Experiment 1 support the idea that an achromatic aftereffect may be generated, since, as this model predicts, highluminance gratings were most efficient at reducing the chromatic effect. Although the outcome of Experiment 2 is not as strongly supportive of this model, it is not contradictory to its predictions. 


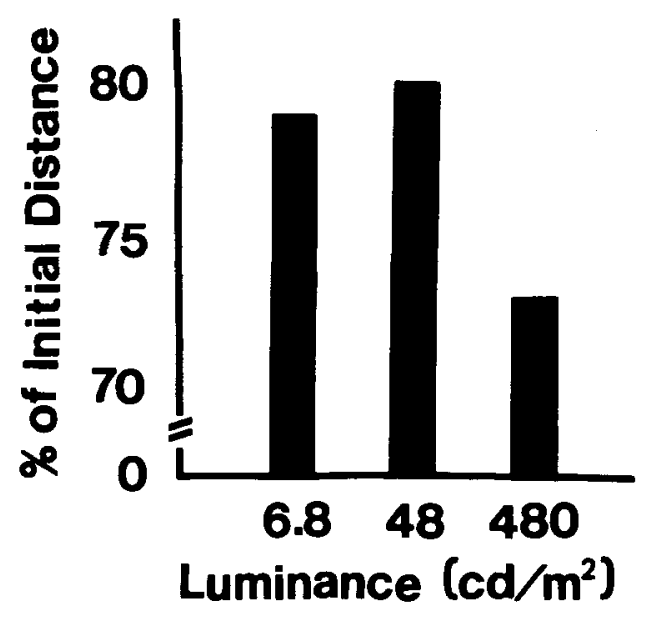

Figure 2. Strength of MEs induced after exposure to achromatic gratings of varying luminance. The ordinate expresses this strength relative to an initial distance between matches typical of each observer under the conditions of Experiment 1. The height of each bar represents an average of three sessions per observer.

The apparent contrast reduction account would probably predict the relative contribution of the three luminances found in Experiment 1, but might not have anticipated that a rather low-luminance pattern could produce a substantial amount of interferencea reduction to some $60 \%$ of the original effects (only about $15 \%$ different, on the average, than a pattern 2 log units higher in luminance). A similar comment might be applied to the results of Experiment 2. While exposure to the achromatic gratings was probably too removed in time to affect the test pattern contrast directly, viewing rather intense achromatic patterns might reduce the apparent contrast of the chromatic patterns, and hence, presumably, reduce the efficiency of these patterns in generating the ME. Again, however, even low-luminance patterns succeeded in altering the strength assessments.

The classical conditioning model is difficult to evaluate, since it seems ambiguous in its predictions. MEs show up best on low-luminance test patterns, so it might be supposed that a low-luminance interference pattern would tap into the mechanism which reveals the effect with greatest efficiency. Therefore, interference (extinction) would be maximal at low luminances. This, of course, was not found in the present study. However, in the conditioning literature, it has been noted that generalized stimuli with intensities greater than the intensity of the conditioned stimulus tend to elicit stronger responses (Hall, 1976). It might be possible to predict, therefore, that a high-intensity extinction stimulus would be most effective. Analogous reasoning could be applied to the results of Experiment 2, if interference in advance of induction is interpreted as an instance of latent inhibition. The problem with this line of reasoning is that the McCollough effect does not conform to the basis for the prediction-appearance and assessment of the effects are always strongest when the stimuli are of substantially less intensity than the conditioned stimulus used to build up the response (White, 1976). This characteristic of MEs is contrary to predictions of a conditioning model.

An explanation of interference phenomena based on the generation of a competing achromatic effect remains extremely speculative, since achromatic analogues of McCollough effects have not been demonstrated independently. Devising a means of directly testing their existence would seem to be the next step in solving this puzzle.

\section{REFERENCE NOTES}

1. White, K. D., \& Graves, A. L. Influence of achromatic inspection on McCollough effects. Paper presented at the meeting of the Association for Research in Vision and Ophthalmology, Sarasota, Florida, May 1976.

2. White, K. D. Personal communication, 1976.

3. White, K. D., \& Graves, A. L. Test pattern contrast influences assessed strengths of McCollough effects. Personal communication with K. D. White, 1977.

\section{REFERENCES}

Hall, J. J. Classical conditioning and instrumental learning. New York: Lippincott, 1976.

Held, R., \& Shattuck, S. Color- and edge-sensitive channels in the human visual system: Tuning for orientation. Science, 1971, 174, 314-316.

Jones, P. D., \& Holding, D. H. Extremely long-term persistence of the McCollough effect. Journal of Experimental Psychology: Human Perception and Performance, 1975, 1, 323-327.

McCollough, C. Color adaptation of edge-detectors in the human visual system. Science, 1965, 149, 1115-1116.

Murch, G. M. Classical conditioning of the McCollough effect: Temporal parameters. Vision Research, 1976, 16, 615-619.

Skowbo, D., \& Clynes, N. Decline and revival of McCollough effects following inspection of achromatic gratings. Perception \& Psychophysics, 1977, 21, 180-182.

Skowbo, D., Gentry, T., Timney, B., \& Morant, R. B. The McCollough effect: Influence of several kinds of visual stimulation on decay rate. Perception \& Psychophysics, 1974, 16, 47-49.

White, K. D. Luminance as a parameter in establishment and testing of the McCollough effect. Vision Research, 1976, 16, 297-302.

(Received for publication January 18, 1979; revision accepted June 5, 1979.) 